\title{
Complex molecules in Wolf-Rayet ring nebula NGC 2359
}

\author{
J. Ricardo Rizzo ${ }^{1,2}$, Jesús Martín-Pintado ${ }^{2}$, and Jean-François Desmurs ${ }^{2}$ \\ ${ }^{1}$ Departamento de Fisica, Universidad Europea CEES, \\ E-28670, Villaviciosa de Odón, España \\ ${ }^{2}$ Observatorio Astronómico Nacional, \\ Aptdo. Correos 1143, E-28800 Alcalá de Henares, España
}

\begin{abstract}
We report the detection and analysis of several molecules arising from the WR nebula NGC 2359 around the WN4 star WR 7 (HD 56925). We have detected $3 \mathrm{~mm}$ and $1 \mathrm{~mm}$ rotational lines from ${ }^{13} \mathrm{CO}, \mathrm{C}^{18} \mathrm{O}, \mathrm{HCO}^{+}, \mathrm{CS}, \mathrm{CN}$ and $\mathrm{HCN}$, and determined lower limits for the SiO. The survey was performed in the southeastern part of the optical nebula, where we previously inferred the presence of dense $\left(10^{4} \mathrm{~cm}^{-3}\right)$ material.
\end{abstract}

\section{Background}

The molecular content of the Wolf-Rayet ring nebula NGC 2359, around the WN4 star WR 7 (HD 56925), has been a subject of research since a couple of years (St-Louis et al. 1998; Rizzo et al. 2001a and 2001b; Cappa et al. 2001). It has been shown that the southeast of NGC 2359 contains molecular gas which would tell us about the dominant physics and chemistry related to massive star evolution. Our previous detection of abundant and warm ammonia might indicate that this region has been shocked by the early phases of WR 7, at least once. This trend was also confirmed by a velocity shift between ${ }^{13} \mathrm{CO}$ and $\mathrm{CO}$ and a high $\mathrm{CO}(2-1)$ to $\mathrm{CO}(1-0)$ ratio (Rizzo et al. 2002, in preparation).

\section{Observations and results}

We performed a high sensitivity, high resolution survey of complex molecules along a strip in the southeast of NGC 2359. The observations were performed in two observing runs during 2001 at the IRAM $30 \mathrm{~m}$ radio telescope, at the position sketched in the left panel of Figure 1, overlaid to a $\mathrm{CO}(2-1)$ map. We have observed $3 \mathrm{~mm}$ and $1 \mathrm{~mm}$ rotational lines from ${ }^{13} \mathrm{CO}, \mathrm{C}^{18} \mathrm{O}, \mathrm{HCO}^{+}, \mathrm{CS}$, $\mathrm{SiO}, \mathrm{CN}$ and $\mathrm{HCN}$.

Table 1 presents the column densities of the detected species. All molecules but $\mathrm{SiO}$ have been detected, mainly at $3 \mathrm{~mm}$. These detections demonstrate that this WR nebula contains extended, high density regions $\left(10^{4} \mathrm{~cm}^{-3}\right)$. The relative abundances of the $\mathrm{CO}$ isotopes is highly variable from one position to another, reflecting possible excitation and opacity effects. The CN:HCN ratio is certainly high (of the order of 10), which might indicate that the role of photodissociation is important even at such large distance to the WR star. A sample of some spectra in positions B, C and D is displayed in Figure 1. 


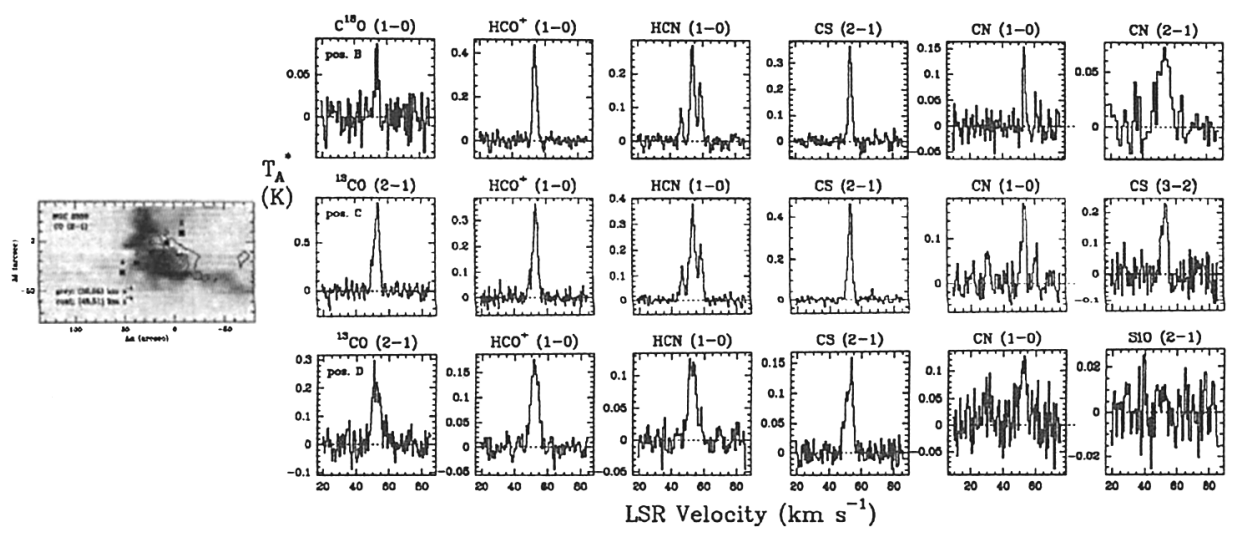

Figure 1. Location of positions (left) and a sample of observed spectra of the Wolf-Rayet ring nebula NGC 2359, around the WN4 star WR 7.

Table 1. Detected molecular species and column densities in NGC 2359.

\begin{tabular}{lllllll}
\hline \hline position & $\mathrm{N}(\mathrm{CO})$ & $\mathrm{N}\left({ }^{13} \mathrm{CO}\right)$ & $\mathrm{N}(\mathrm{CS})$ & $\mathrm{N}\left(\mathrm{HCO}^{+}\right)$ & $\mathrm{N}(\mathrm{CN})$ & $\mathrm{N}(\mathrm{HCN})$ \\
\hline $\mathrm{A}$ & $6.8(15)$ & $9.8(14)$ & $1.4(12)$ & $9.6(11)$ & $<5.4(12)$ & $1.5(11)$ \\
$\mathrm{B}$ & $2.7(16)$ & $3.2(15)$ & $8.0(12)$ & $2.6(12)$ & $4.6(12)$ & $7.2(11)$ \\
$\mathrm{C}$ & $2.7(16)$ & $1.8(15)$ & $7.4(12)$ & $1.1(12)$ & $4.4(12)$ & $3.6(11)$ \\
$\mathrm{D}$ & $2.1(16)$ & $1.3(15)$ & $1.2(13)$ & $1.7(12)$ & $7.7(12)$ & $5.9(11)$ \\
E & $3.4(15)$ & $1.4(14)$ & $1.5(12)$ & $4.8(11)$ & $<2.6(12)$ & $2.3(11)$ \\
\hline \hline
\end{tabular}

Note: numbers in parentheses refer to powers of $10\left(\right.$ in $\left.\mathrm{cm}^{-2}\right)$.

\section{Summary and future work}

The southeast of NGC 2359 has an interesting mixing of photo-dissociation and shocks. A deep knowledge of the physics and chemistry of these processes will help us to understand in detail some aspects of the massive star evolution, such as the existence of episodes of mass ejection and wind variability.

Further observations in $1 \mathrm{~mm}$ lines would tell us about the excitation conditions of these molecules. The extension of this observational effort to other objects - such as WR nebulae with well-known mass ejection, or LBV nebulae - is worth carrying out, in order to get a better determination of the interplay between massive evolved stars and the ISM.

\section{References}

Cappa, C.E., Rubio, M., Goss, W.M. 2001, AJ 121, 2664

Rizzo, J.R., Martín-Pintado, J., Mangum, J.G. 2001a, A\&A 366, 146

Rizzo, J.R., Martín-Pintado, J., Henkel, C. 2001b, ApJ (Letters) 553, L181

St-Louis, N., Doyon, R., Chagnon, F., Nadeau, D. 1998, AJ 115, 2475 